\title{
Multidimensional Poverty and Material Deprivation with Discrete Data*
}

\author{
WALTER BOSSERT \\ Department of Economics and CIREQ, University of Montreal \\ walter.bossert@umontreal.ca \\ SAtya R. Chakravarty \\ Indian Statistical Institute, Kolkata \\ satya@isical.ac.in \\ Conchita D'Ambrosio \\ Università di Milano-Bicocca, DIW Berlin and Econpubblica \\ conchita.dambrosio@gmail.com
}

* We thank two referees for useful suggestions as well as the Fondazione Debenedetti for providing access to the dataset. Financial support from the Social Sciences and Humanities Research Council of Canada is gratefully acknowledged. 


\begin{abstract}
We propose a characterization of a popular index of multidimensional poverty which, as a special case, generates a measure of material deprivation. This index is the weighted sum of the functioning failures. The important feature of the variables that may be relevant for poverty assessments is that they are discrete in nature. Thus, poverty measures based on continuous variables are not suitable in this setting and the assumption of a discrete domain is mandatory. We apply the measure to European Union member states where the concept of material deprivation was initiated and illustrate how its recommendations differ from those obtained from poverty measures based exclusively on income considerations. Journal of Economic Literature Classification No.: D63.
\end{abstract}

Keywords: Multidimensional Poverty Measurement, Material Deprivation, Counting Approach. 


\section{Introduction}

An important development in the study of inequality and poverty in the recent past is the shift of emphasis from a single dimension, such as income, to a multidimensional framework. There are several reasons for this phenomenon.

First, contributions such as those of Townsend (1979), Streeten (1981) and Sen (1992) highlighted that the well-being of an individual, and hence the inequality and poverty in a population, is dependent on many dimensions of human life such as housing, education and life expectancy; income is but one of these dimensions. Thus, poverty may be better defined as a situation that reflects failures in different dimensions of human wellbeing. The multidimensionality of an individual's well-being has also been emphasized by the Commission on the Measurement of Economic Performance and Social Progress; see Stiglitz, Sen and Fitoussi (2009, p.14).

Second, in the income distribution literature, income is not important per se but is supposed to be an indicator of an individual's command over economic resources. But income may not always be suitable for this purpose because it neglects command over resources out of wealth, non-cash transfers from the government and support from family and friends; see also, for instance, Ringen (1988). Thus, to measure command over economic resources, aspects other than income should be included. In doing so, it is necessary to distinguish the absence of consumption due to individual preferences from the absence of consumption due to inability to afford. Obviously, the former should not be considered in measuring poverty. In addition, for policy purposes, it is necessary to identify the fragment of the population who is currently poor. In a typical dataset, the information on income received refers to the previous calendar year (and is more likely to be misreported - particularly, underreported) while items of consumption are reported contemporaneously.

The third reason is of great importance for the European Union, where a shift in policy focus from pure income poverty towards a wider multidimensional framework has been particularly pronounced. Changes in public policies implemented by the member states were initiated at the March 2000 Lisbon European Council. At this Council, the member states agreed to adopt the Open Method of Coordination which involves the definition of a set of common objectives on poverty and social exclusion for the EU as a whole. The successor of the Lisbon Agenda is the Europe 2020 strategy of growth: the EU has set five objectives - on employment, innovation, education, social inclusion and climate/energyto be reached by 2020. The EU distinguishes itself from other political entities in that 
it clearly endorses the use of relative poverty lines. The measures of income poverty adopted are based on member-specific poverty lines, that is, for each member state, the income threshold depends on the income distribution of the specific country and does not take into account inequality between member states. This practice has become more problematic with the enlargement of the Union and the substantial differences that can be observed between the income distributions of old and new members. Someone poor in one of the old member states is likely to be located well above average in the income distribution of a new member state. Should the poor member states be taxed and the rich countries receive a transfer in response? This clearly would be an absurd recommendation. It appears evident that the development of other indicators of an individual's command over economic resources is desirable. For a discussion of this point in the EU context, see, among others, Fahey (2007) and Whelan, Nolan and Maitre (2008).

The distinction between multidimensional poverty and material deprivation we use is that endorsed by the EU. In particular, a multidimensional poverty measure takes into consideration all dimensions of well-being that may be of relevance (including nonmaterial attributes such as health status and political participation), whereas an index of material deprivation restricts attention to functioning failures regarding material living conditions (see, among others, Guio, 2005). According to EU policy, indices of material deprivation are to be combined with income-based poverty measures and indicators of low employment. This paper constitutes an attempt to contribute to this objective.

The purpose of this paper is to characterize a popular measure of multidimensional poverty and use it to evaluate material deprivation in the EU. This index is the weighted sum of the functioning failures. An important feature of the various variables that may be relevant for poverty assessments is that they are discrete in nature, that is, what is considered relevant for a person (and what appears in the data) is whether or not a functioning failure with respect to the dimension under consideration obtains. Thus, poverty measures based on continuous variables are not be suitable in this setting and the assumption of a discrete domain is mandatory. This distinction is usually referred to as qualitative/ordinal versus quantitative/cardinal variables; however, because we identify a functioning failure with a value of one and the absence of a functioning failure with a value of zero for the requisite variable, we prefer to use the terms discrete and continuous instead.

The importance of the ability to deal with discrete data is that, usually, only very few of a survey's variables on individual well-being are continuous in nature. This situation is common to many surveys; see, for example, the European Community Household Panel 
or the more recent EU Statistics on Income and Living Conditions (EU-SILC) for EU countries, and the United States' Current Population Survey, where most of the variables that may be used to measure multidimensional poverty are discrete. Hence, most of the indices proposed in the literature dealing with continuous variables cannot be applied. An alternative is what Atkinson (2003) refers to as the counting approach. The counting measure of individual poverty consists of the number of dimensions in which a person is poor, that is, the number of the individual functioning failures. But this measure treats all dimensions symmetrically in the sense that in the aggregation of an individual's functioning failures, the same weight is assigned to each dimension. Since some of the dimensions may be more important than others, a more appropriate counting measure can be obtained by assigning different weights to different dimensions and then adding these weights for the dimensions in which functioning failure is observed. These weights may be assumed to reflect the importance a policy maker attaches to alternative dimensions in a povertyalleviation proposal. For instance, for evaluating multidimensional poverty in Mexico, Foster (2007) assumed a weight structure which first splits weights between income and non-income dimensions equally and then uses equal weights for non-income dimensions. Alternatively, the weights may reflect views of the society under analysis which is the approach followed in the present contribution. This weighting scheme is known in the EU political debate as consensus weighting. As opposed to the prevalence or frequency-based weighting, it has the advantage of better reflecting the minimum acceptable standard of living, which is what material deprivation indicators aim to capture. For a discussion of weighting schemes in EU indicators, see Guio, Fusco and Marlier (2009). A survey on the use of weights in multidimensional indices of well-being can be found in Decancq and Lugo (2011).

The shift of emphasis towards multidimensionality has raised many challenges for social scientists interested in measuring poverty in well-being. The two-stage procedure suggested by Sen (1976) - consisting of first identifying the poor and then aggregating the information available on this segment of the population into an index of poverty for the entire society — has to be extended.

In the multidimensional framework, each person is assigned a vector of several attributes that represent different dimensions of well-being. For measuring multidimensional poverty, it then becomes necessary to check whether a person has "minimally acceptable levels" of these attributes; see Sen (1992, p.139). These minimally acceptable quantities of the attributes represent their threshold limits or cut-offs that are necessary for an adequate standard of living. Therefore, a person is treated as deprived or poor in a 
dimension if the requisite observed level falls below this cut-off. In this case we say that the individual is experiencing a functioning failure. Poverty at the individual level is an increasing function of these failures.

The first stage consisting of the identification of the poor in a multivariate framework is still an issue subject to debate. One possible way of regarding a person as poor is if the individual experiences a functioning failure in every dimension, which identifies the poor as those who are poor in all dimensions. This is known as the intersection method of identification of the poor. But if a person is poor in one dimension and non-poor in another, then trading off between the two dimensions may not be possible. Lack of access to essential durables, say, cannot be compensated by housing. In view of this, a person may be treated as poor if she is poor in at least one dimension. This is the union method of identifying the poor; see Tsui (2002) and Bourguignon and Chakravarty (2003). In between these two extremes lies the intermediate identification method which regards a person as poor if she is deprived in at least $m \in\{1, \ldots, K\}$ dimensions, where $K$ is the number of dimensions on which human well-being depends. See Mack and Lindsay (1985), Gordon, Nandy, Pantazis, Pemberton and Townsend (2003) and Alkire and Foster (2011). Evidently, the intermediate method contains the union and the intersection methods as special cases for $m=1$ and $m=K$, respectively. Our approach to identification follows the union method: a person is considered poor if she is poor in at least one dimension.

The axiomatic literature on the subject has proposed some measures of multidimensional poverty and explored the properties that are at the basis of these indices; see, for example, Chakravarty, Mukherjee and Ranade (1998), Tsui (2002), Bourguignon and Chakravarty (2003), Diez, Lasso de la Vega and Urrutia (2008) and Alkire and Foster (2011). However, with the exception of Alkire and Foster (2011), the functionings considered in these contributions are expressed by means of continuous variables. Alkire and Foster's (2011) index is for discrete data but no characterization has been provided by the authors. Hence, to the best of our knowledge, our approach is novel in this respect. Another important contribution on multidimensional poverty with discrete variables is that of Lasso de la Vega (2010), where counting poverty orderings and deprivation curves are proposed.

The remainder of the paper proceeds as follows. We characterize the index of multidimensional poverty that allows for the assignment of different weights to the considered dimensions in section 2, and we apply this measure to illustrate its use in assessing material deprivation in the European Union using the EU-SILC dataset in section 3. Section 4 provides some brief concluding remarks. 


\section{The Index of Multidimensional Poverty}

Suppose there are $K \in \mathbb{N} \backslash\{1\}$ dimensions that may be relevant for the degree of wellbeing of an individual, such as housing conditions. These dimensions are the same across societies and they are represented by binary variables: a value of one indicates that the individual is poor with respect to this dimension, a value of zero identifies a attribute with respect to which the individual is not poor. Throughout, the number of relevant dimensions is assumed to be fixed. We adopt the union method of identifying the poor in the sense that a person is considered poor if she is poor in at least one of the relevant dimensions.

In order to be applied to different societies or to different time periods, a suitable measure of poverty must be capable of accommodating different population sizes. Thus, we consider all possible population sizes $N \in \mathbb{N}$ when defining a measure of multidimensional poverty. Let $N \in \mathbb{N}$ and $K \in \mathbb{N} \backslash\{1\}$. A dichotomous $N \times K$ matrix is a matrix

$$
M=\left(m_{n}^{k}\right)_{\substack{n \in\{1, \ldots, N\} \\ k \in\{1, \ldots, K\}}}
$$

such that $m_{n}^{k} \in\{0,1\}$ for all $n \in\{1, \ldots, N\}$ and for all $k \in\{1, \ldots, K\}$. The rows of $M$ correspond to the members of society, the columns represent the attributes considered relevant for poverty measurement. If $m_{n}^{k}=1$, individual $n \in\{1, \ldots, N\}$ is poor with respect to attribute $k \in\{1, \ldots, K\}$ and if $m_{n}^{k}=0$, the person is not poor with respect to this dimension. For $N \in \mathbb{N}$, let $\mathcal{M}_{N}$ be the set of all dichotomous $N \times K$ matrices. The number of attributes $K$ is suppressed in this definition because it is assumed to be fixed. For $N \in \mathbb{N}, n \in\{1, \ldots, N\}$ and $k \in\{1, \ldots, K\}$, we write $m_{n}$ for the $1 \times K$ matrix consisting of the $n^{\text {th }}$ row and $m^{k}$ for the $N \times 1$ matrix consisting of the $k^{\text {th }}$ column of $M \in \mathcal{M}_{N}$.

Define $\mathcal{M}=\cup_{N \in \mathbb{N}} \mathcal{M}_{N}$. An anonymous multidimensional poverty measure is a function $P: \mathcal{M} \rightarrow \mathbb{R}$ such that, for all $M \in \mathcal{M}, P$ is invariant with respect to row permutations of $M$-that is, $P$ is anonymous in the sense that $P$ treats individuals symmetrically, paying no attention to the labels that we may assign to them.

We now formulate the properties that we require $P$ to possess. To do so, we introduce some more notation. For $N \in \mathbb{N}$, let $\mathbf{0}_{N}$ be the $N \times K$ matrix all of whose entries are equal to zero. For $k \in\{1, \ldots, K\}$, let $\mathbf{1}^{k}$ be the $1 \times K$ matrix with $m_{1}^{k}=1$ and $m_{1}^{j}=0$ for all $j \in\{1, \ldots, K\} \backslash\{k\}$. In order to keep our exposition simple, we adopt the convention

$$
\sum_{k \in \emptyset} \alpha^{k}=0
$$


The first two axioms are limited in scope because they apply to one-person societies only.

Zero normalization. For all $M \in \mathcal{M}_{1} \backslash\left\{\mathbf{0}_{1}\right\}$,

$$
P(M)>P\left(\mathbf{0}_{1}\right)=0
$$

This normalization assumption is standard: if the individual in a one-person society is not poor in any attribute, we require the value of the index to be zero and if she is poor in at least one dimension, the index assumes a positive value. Note that this property is based on a union identification of the poor.

Additive decomposability in attributes. For all $M, M^{\prime} \in \mathcal{M}_{1}$ such that $\left(M+M^{\prime}\right) \in$ $\mathcal{M}_{1}$

$$
P\left(M+M^{\prime}\right)=P(M)+P\left(M^{\prime}\right) .
$$

Additive decomposability in attributes is straightforward as well and has been employed in numerous contributions in the field of social index numbers. Sometimes a non-additive formulation may generate problems which do not arise with additively decomposable measures. For instance, in the (non-additive) human development index, if attainment in one of the dimensions approaches its minimum value, this index approaches zero no matter what values are assumed in the other dimensions. This problem can be avoided under an additively decomposable structure; see Ravallion (2011, 2012).

Additive decomposability in attributes entails a separability property: the contribution of any variable to the overall index value can be examined in isolation, without having to know the values of the other variables. Thus, additive decomposability properties are often linked to independence conditions of various forms. Note that, because of the discrete domain considered here, an independence condition is not sufficient unless there are at most four dimensions to poverty; this can be seen by adapting the corresponding result in Kraft, Pratt and Seidenberg (1959) to our setting. Because we work with a general number of poverty attributes and, moreover, the data set used in our application covers more than four attributes, the full force of additive decomposability in attributes is required in our characterization.

We are well aware that additive decomposability is a strong property and that it is no surprise that the resulting index is additive. However, given that our objective is the characterization of a known additive measure, an additivity property cannot but appear in the list of requisite axioms. Given the prominent role played by this measure and the 
absence of a characterization on a discrete domain in the existing literature, it seems to us that this is an appropriate way to proceed. It may be worthwhile to note that variants of the continuous counterpart to the additive decomposability postulate were used earlier in the literature. Chakravarty, Mukherjee and Ranade (1998) used one form of this axiom along with subgroup decomposability to characterize the multidimensional poverty indices that are both factor and subgroup decomposable. Alkire and Foster (2011) noted that given the identification step, total poverty according to their index can be regarded as a weighted average of dimensional values. For a characterization of an additive measure of social exclusion on a discrete domain, see Chakravarty and D'Ambrosio (2006). Jayaraj and Subramanian (2010) apply this index to measure deprivation in India.

As a preliminary result, we identify the class of measures that satisfy the above axioms. Clearly, due to the restriction to one-person societies in these properties, all that can be deduced at this stage is the structure of $P$ on the subdomain of dichotomous matrices with a single row only.

Lemma 1 If an anonymous multidimensional poverty measure $P$ satisfies zero normalization and additive decomposability in attributes, then there exists a vector of parameters $\alpha=\left(\alpha^{1}, \ldots, \alpha^{K}\right) \in \mathbb{R}_{++}^{K}$ such that, for all $M \in \mathcal{M}_{1}$,

$$
P(M)=\sum_{\substack{k \in\{1, \ldots, K\}: \\ m_{1}^{k}=1}} \alpha^{k}
$$

Proof. Suppose $P$ satisfies zero normalization and additive decomposability in attributes. That $P\left(\mathbf{0}_{1}\right)=0$ follows immediately from the equality in zero normalization. Now suppose $M \in \mathcal{M}_{1} \backslash\left\{\mathbf{0}_{1}\right\}$. Define, for all $k \in\{1, \ldots, K\}, \alpha^{k}=P\left(\mathbf{1}^{k}\right)$. By the inequality in the definition of zero normalization, it follows that $\alpha^{k}>0$ for all $k \in\{1, \ldots, K\}$. Writing $M$ as

$$
M=\sum_{\substack{k \in\{1, \ldots, K\}: \\ m_{1}^{k}=1}} \mathbf{1}^{k},
$$

additive decomposability in attributes requires

$$
P(M)=\sum_{\substack{k \in\{1, \ldots, K\}: \\ m_{1}^{k}=1}} P\left(\mathbf{1}^{k}\right)=\sum_{\substack{k \in\{1, \ldots, K\}: \\ m_{1}^{k}=1}} \alpha^{k}
$$

which completes the proof. 
The real number $\alpha^{j}$ is an indicator of the importance that we assign to dimension $j$ when a person is found to be deprived in this dimension. It can as well be interpreted as the priority assigned by the government to remove deprivation in dimension $j$. The index $P(M)$ is simply the total of such indicators across dimensions.

The last axiom used in our characterization parallels the above additive decomposability property with respect to attributes. We require that $P$ be additively decomposable in individuals as well, with suitable weights applied so as to take proper account of population size. Clearly, as is the case for unidimensional poverty measures, the total number of individuals matters. Consider a society $A$ in which one hundred out of a thousand people are poor. Furthermore, suppose a society $B$ is such that, again, one hundred people are poor (to the same degree as the poor in $A$ ) but total population size is one million in $B$. All poverty measures usually employed assign a higher level of poverty to $A$ than to $B$, which reflects the view that poverty is a per-capita notion. Thus, we formulate our third axiom as follows.

Population-weighted additive decomposability in individuals. For all $N \in \mathbb{N}$, for all $M^{\prime} \in \mathcal{M}_{N}$, for all $M^{\prime \prime} \in \mathcal{M}_{1}$ and for all $M \in \mathcal{M}_{N+1}$, if $m_{n}=m_{n}^{\prime}$ for all $n \in\{1, \ldots, N\}$ and $m_{N+1}=m_{1}^{\prime \prime}$, then

$$
P(M)=\frac{N}{N+1} P\left(M^{\prime}\right)+\frac{1}{N+1} P\left(M^{\prime \prime}\right) .
$$

See our earlier discussion of additive decomposability in attributes for a motivation of this decomposability property. Again, a property of this nature is required given that we aim at characterizing a measure with an additive structure on a discrete domain.

We obtain

Theorem 1 An anonymous multidimensional poverty measure $P$ satisfies zero normalization, additive decomposability in attributes and population-weighted additive decomposability in individuals if and only if there exists a vector of parameters $\alpha=\left(\alpha^{1}, \ldots, \alpha^{K}\right) \in$ $\mathbb{R}_{++}^{K}$ such that, for all $N \in \mathbb{N}$ and for all $M \in \mathcal{M}_{N}$,

$$
P(M)=\frac{1}{N} \sum_{n=1}^{N} \sum_{\substack{k \in\{1, \ldots, K\}: \\ m_{n}^{k}=1}} \alpha^{k}
$$

Proof. The 'if' part of the theorem statement is straightforward to verify. To prove the 'only if' part, we proceed by induction on the population size. Suppose that $P$ satisfies 
the required axioms. Lemma 1 establishes the claim for all $M \in \mathcal{M}_{1}$. Now suppose (1) is true for all population sizes from one to $N \in \mathbb{N}$. Let

$$
\begin{gathered}
M \in \mathcal{M}_{N+1}, \\
M^{\prime}=\left(m_{n}^{k}\right)_{\substack{n \in\{1, \ldots, N\} \\
k \in\{1, \ldots, K\}}}, \\
M^{\prime \prime}=m_{N+1} .
\end{gathered}
$$

By population-weighted additive decomposability in individuals and our induction hypothesis, it follows that

$$
\begin{aligned}
P(M) & =\frac{N}{N+1} P\left(M^{\prime}\right)+\frac{1}{N+1} P\left(M^{\prime \prime}\right) \\
& =\left(\frac{N}{N+1}\right) \frac{1}{N} \sum_{n=1}^{N} \sum_{\substack{k \in\{1, \ldots, K\}: \\
m_{n}^{k}=1}} \alpha^{k}+\frac{1}{N+1} \sum_{\substack{k \in\{1, \ldots, K\}: \\
m_{N+1}^{k}=1}} \alpha^{k} \\
& =\frac{1}{N+1} \sum_{n=1}^{N+1} \sum_{\substack{k \in\{1, \ldots, K\}: \\
m_{n}^{k}=1}} \alpha^{k}
\end{aligned}
$$

where we used the anonymity assumption on $P$ to deduce that the parameters $\alpha^{k}$ do not depend on the labels of the individuals under consideration.

It may be noted that while Bourguignon and Chakravarty (2003) used the deprivation count, the number of dimensions of well-being from which a person is deprived in the union/intersection framework for identification of the poor, the Alkire and Foster (2011) identification method relies on the counting formula using unequal weighting for dimensions in the intermediate set up. Lasso de la Vega (2010) examined dominance conditions for poverty orderings using the counting approach based on this identification method. Aaberge and Peluso (2011) compared deprivation counts of distributions using rank dependent social evaluation criteria. This clearly indicates that Theorem 1 has different objectives than the counting based results reported in the above papers.

Note that we do not employ a focus axiom analogous to that familiar from unidimensional poverty measurement. This is the case because our (union) identification of the poor is implicit in our axioms - the poor are those who experience a functioning failure in at least one dimension, and the characteristics of the non-poor (those who do not experience any functioning failure) do not influence the value of the index. 


\section{Material Deprivation within the EU}

In this section, we illustrate the index defined in (1) by employing it to the problem of measuring material deprivation in the EU. Recall that, in assessing material deprivation as opposed to multidimensional poverty in general, we focus on dimensions that represent access to material economic resources. The dataset we use is EU-SILC, which is employed by European Union member states and the Commission to monitor national and EU progress towards key objectives for the social inclusion process and Europe 2020 growth strategy. Our analysis is for the years from 2005 to 2008. The variables that may be used in the measurement of material deprivation are available mainly at the household level. We follow a conservative approach in the sense that we treat the households reporting a missing value like those reporting not to experience the functioning failure. As a result, we may be underestimating material deprivation since we are attributing a functioning failure exclusively to households who explicitly claim to have the failure. We also perform some sensitivity analysis and excluded the missing values from the sample. The results do not change, hence they are omitted but are available upon request. The unit of our analysis is the individual, that is, the household failure is attributed to each household member and we analyze the distribution of functioning failures among individuals.

In line with the Europe 2020 framework, the variables we consider are the following.

1. The household has been in arrears at any time in the last 12 months on mortgage or rent payments.

2. The household has been in arrears at any time in the last 12 months on utility bills.

3. The household lacks the ability to keep the home adequately warm.

4. The household lacks the capacity to face unexpected required expenses.

5. The household cannot afford a meal with meat, chicken, fish (or a vegetarian protein equivalent) every second day.

6. The household cannot afford to pay for a one-week annual holiday away from home.

7. The household cannot afford to have a car.

8. The household cannot afford a washing machine.

9. The household cannot afford a color TV. 
10. The household cannot afford a telephone.

The weights are constructed from the views of EU citizens as surveyed in 2007 in the special Eurobarometer 279 on poverty and social exclusion (see TNS Opinion \& Social, 2007). This weighting method has first been proposed by Guio, Fusco and Marlier (2009). For each variable, we use as weight the percentage of the EU27 citizens answering "absolutely necessary, no one should have to do without" to the requisite question as expressed by these instructions: "In the following questions, we would like to understand better what, in your view, is necessary for people to have what can be considered as an acceptable or decent standard of living in (OUR COUNTRY). For a person to have a decent standard of living in (OUR COUNTRY), please tell me how necessary do you think it is ... (if one wants to)." The possible answers also included "necessary," "desirable but not necessary" and "not at all necessary." The answers given by citizens living in EU27 are reported in Table 1. The weights we use constitute the entries in column 2. They range from $68 \%$ for the absolutely necessity of not being in arrears on utility bills to $17 \%$ for the absolutely necessity of affordability of a car. We compare our results (using the ten discrete variables introduced above) with those obtained by weighting all functioning failures equally, and with those according to the (solely income-based) head-count ratio with the 60-per-cent-of-the-median-equivalent-income country-specific poverty lines.

The results of our analysis are summarized in Table 2. The first column lists the official abbreviation of country names, whereas the second set of columns contains the rankings for the four years obtained according to the headcount ratio on household equivalent income. The remaining two sets of columns include the values of the material deprivation index defined in (1) for the various years, the first four with the Eurobarometer weights, the other four when equal weight is given to each dimension. The performance of the countries over time is more stable for material deprivation than for income poverty as measured by the head-count ratio. The results are sensitive to the choice of the weights for some of the countries such as Austria, Estonia, Iceland and Spain. Iceland's position improves by five when equal weighting is given to all dimensions in 2005, three in the next two years and two positions in 2008. Estonia moves down in the rankings by four positions in 2005 and 2008 and by three in the two other years.

\section{Insert Tables here}

In Figures 1 and 2 we plot, for each year, the rankings of material deprivation with respect to the head-count ratio. A very different picture emerges when comparing the 
performance of the countries depending on whether we look at income poverty (measured by the head-count ratio) or at material deprivation, confirming that these two phenomena differ considerably among European countries. For similar findings, see, among others, Guio, Fusco and Marlier (2009) and Whelan and Maître (2009). We observe a decrease in the rankings of old EU member states, where a substantial level of material living conditions has been reached, and a worsening of the position of new member states, with few exceptions. Ireland, Luxembourg, the UK and Spain are the countries which considerably improve their position in all of the years, whereas for the Republic of Cyprus, the Czech Republic, Hungary and Slovakia, we observe the reverse phenomenon. Slovenia belongs to the latter group only for the first three years of the analysis. The highest material deprivation rates are exhibited by the new EU member states, where income poverty is low due to a narrow income distribution.

\section{Insert Figures here}

These basic findings suggest that European social policy aiming at assisting citizens with low well-being may be better performed by combining information on income poverty and material deprivation: indicators based solely on income poverty do not appear to be sufficient to capture living conditions adequately. Since the EU endorses the use of relative poverty lines, the absolute component of well-being is considered with measures of material deprivation.

\section{Concluding Remarks}

In this paper we provide an axiomatic characterization of a popular index of multidimensional poverty, the weighted sum of the functioning failures, and apply it to assess material deprivation in the EU. The novelty of the theoretical approach is that the characterization applies to a discrete domain where standard techniques used on a continuum cannot be applied. The measure resembles Bourguignon and Chakravarty's (2003) index for measuring multidimensional poverty in the case of continuous variables. An interesting possibility for future research is to provide a characterization of an index based on both continuous and discrete data. This index may also consider the degree of dependence between attributes, an issue that has attracted increasing attention in the study of multidimensional well-being. 


\section{References}

Aaberge, R. and E. Peluso, "A Counting Approach for Measuring Multidimensional Deprivation," Università di Verona, Dipartimento di Economia, WP No.7, 2011.

Alkire, S. and J.E. Foster, "Counting and Multidimensional Poverty Measurement," Journal of Public Economics, 95, 476-487, 2011.

Atkinson, A.B., "Multidimensional Deprivation: Contrasting Social Welfare and Counting Approaches," Journal of Economic Inequality, 1, 51-65, 2003.

Bourguignon, F. and S.R. Chakravarty, "The Measurement of Multidimensional Poverty," Journal of Economic Inequality, 1, 25-49, 2003.

Chakravarty, S.R., D. Mukherjee and R. Ranade, "On the Family of Subgroup and Factor Decomposable Measures of Multidimensional Poverty," Research on Economic Inequality, 8, 175-194, 1998.

Chakravarty, S.R. and C. D'Ambrosio, "The Measurement of Social Exclusion," Review of Income and Wealth, 52, 377-398, 2006.

Decancq, K. and M.A. Lugo, "Weights in Multidimensional Indices of Well-Being: An Overview," Econometric Reviews, forthcoming, 2011.

Diez, H., M.C. Lasso de la Vega and A.M. Urrutia, "Multidimensional Unit- and Subgroup Consistent Inequality and Poverty Measures: Some Characterization Results," Research on Economic Inequality, 16, 189-211, 2008.

Fahey, T., "The Case for an EU-wide Measure of Poverty," European Sociological Review, $23,35-47,2007$.

Foster, J.E., "Report on Multidimensional Poverty Measurement," El Colegio de Mexico, Mexico City, 2007.

Gordon, G., S. Nandy, C. Pantazis, S. Pemberton and P. Townsend, Child Poverty in the Developing World, The Policy Press, Bristol, 2003.

Guio, A.-C., "Material Deprivation in the EU," Statistics in Focus, Population and Social Conditions, Living Conditions and Welfare, 21/2005, Eurostat, Luxembourg, 2005.

Guio, A.-C., A. Fusco and E. Marlier, "An EU Approach to Material Deprivation using EU-SILC and Eurobarometer Data," IRISS Working Paper 2009-19, 2009.

Jayaraj, D. and S. Subramanian, "A Chakravarty-DAmbrosio View of Multidimensional Deprivation: Some Estimates for India," Economic and Political Weekly, 45, 53-65, 2010. 
Kraft, C.H., J.W. Pratt and A. Seidenberg, "Intuitive Probability on Finite Sets," Annals of Mathematical Statistics, 30, 408-419, 1959.

Lasso de la Vega, M.C., "Counting Poverty Orderings and Deprivation Curves," Research on Economic Inequality, 18, 153-172 2010.

Mack, J. and S. Lindsay, Poor Britain, George Allen and Unwin Ltd., London, 1985.

Ravallion, M., "The Human Development Index: A Comment on Klugman, Rodriguez and Choi," Journal of Economic Inequality, 9, 475-478, 2011.

Ravallion, M., "Troubling Tradeoffs in the Human Development Index," Journal of Development Economics, forthcoming, 2012.

Ringen, S., "Direct and Indirect Measures of Poverty," Journal of Social Policy, 17, 351$365,1988$.

Sen, A., "Poverty: An Ordinal Approach to Measurement," Econometrica, 44, 219-231, 1976.

Sen, A., Inequality Re-examined, Harvard University Press, Cambridge, MA, 1992.

Stiglitz, J.E., A. Sen and J.-P. Fitoussi, Report by the Commission on the Measurement of Economic Performance and Social Progress, CMEPSP, 2009.

Streeten, P., First Things First: Meeting Basic Human Needs in Developing Countries, Oxford University Press, New York, 1981.

TNS Opinion \& Social, "Poverty and Exclusion," Report on the Special Eurobarometer No. 279/Wave 67.1, available at:

http://ec.europa.eu/public_opinion/archives/ebs/ebs_279.pdf, 2007.

Townsend, P., Poverty in the United Kingdom, Penguin, Harmondsworth, 1979.

Tsui, K.-Y., "Multidimensional Poverty Indices," Social Choice and Welfare, 19, 69-93, 2002 .

Whelan, C.T. and B. Maître, "Europeanization of Inequality and European Reference Groups," Journal of European Social Policy, 19, 117-130, 2009.

Whelan, C.T., B. Nolan and B. Maître, "Measuring Material Deprivation in the Enlarged EU," ESRI Working Paper No.249, 2008. 


\section{Tables}

Table 1: Answers in percentages to: "In the following questions, we would like to understand better what, in your view, is necessary for people to have what can be considered as an acceptable or decent standard of living in (OUR COUNTRY). For a person to have a decent standard of living in (OUR COUNTRY), please tell me how necessary do you think it is... (if one wants to)".

\begin{tabular}{|c|c|c|c|c|}
\hline EU27 & $\begin{array}{c}\text { Absolutely } \\
\text { necessary, no } \\
\text { one should have } \\
\text { to do without } \\
\end{array}$ & Necessary & $\begin{array}{c}\text { Desirable } \\
\text { but not } \\
\text { necessary }\end{array}$ & $\begin{array}{c}\text { Not at all } \\
\text { necessary }\end{array}$ \\
\hline A place to live without a leaking roof, damp walls, floors, foundation & $68 \%$ & $28 \%$ & $3 \%$ & $1 \%$ \\
\hline To be able to keep one's home adequately warm & $62 \%$ & $35 \%$ & $3 \%$ & $0 \%$ \\
\hline A place to live with its own bath or shower & $63 \%$ & $31 \%$ & $6 \%$ & $0 \%$ \\
\hline An indoor flushing toilet for sole use of the household & $69 \%$ & $27 \%$ & $4 \%$ & $0 \%$ \\
\hline To be able to pay rent or mortgage payments on time & $62 \%$ & $34 \%$ & $3 \%$ & $0 \%$ \\
\hline To be able to pay utility bills (electricity, water, gas, etc.) on time & $68 \%$ & $30 \%$ & $2 \%$ & $0 \%$ \\
\hline $\begin{array}{l}\text { To be able to repay loans (such as loans to buy electrical appliances, furniture, a car or student } \\
\text { loans, etc.) on time }\end{array}$ & $48 \%$ & $40 \%$ & $9 \%$ & $2 \%$ \\
\hline Paying for one week annual holiday away from home & $15 \%$ & $29 \%$ & $43 \%$ & $13 \%$ \\
\hline A meal with meat, chicken or fish at least once every two days & $43 \%$ & $37 \%$ & $17 \%$ & $3 \%$ \\
\hline To be able to cope with an unexpected financial expense of X (NATIONAL CURRENCY) & $32 \%$ & $43 \%$ & $21 \%$ & $2 \%$ \\
\hline A fixed telephone, landline & $18 \%$ & $37 \%$ & $32 \%$ & $13 \%$ \\
\hline A mobile phone & $12 \%$ & $26 \%$ & $37 \%$ & $25 \%$ \\
\hline A colour TV & $19 \%$ & $36 \%$ & $35 \%$ & $10 \%$ \\
\hline A computer & $9 \%$ & $21 \%$ & $41 \%$ & $28 \%$ \\
\hline A washing machine & $48 \%$ & $41 \%$ & $10 \%$ & $1 \%$ \\
\hline A car & $17 \%$ & $34 \%$ & $36 \%$ & $13 \%$ \\
\hline $\begin{array}{l}\text { A place to live without too much noise from neighbours or noise from the street (traffic, businesses, } \\
\text { factories, etc.) }\end{array}$ & $28 \%$ & $43 \%$ & $27 \%$ & $2 \%$ \\
\hline $\begin{array}{l}\text { A place to live without too much pollution or other environmental problems (such as air pollution, } \\
\text { grime or rubbish) }\end{array}$ & $42 \%$ & $44 \%$ & $13 \%$ & $1 \%$ \\
\hline A place to live without crime, violence or vandalism in the area & $49 \%$ & $38 \%$ & $12 \%$ & $1 \%$ \\
\hline
\end{tabular}


Table 2: Material Deprivation (MD) and Income Poverty (H) Ranks among EU Member States in the years 2005-2008, with Eurobarometer Weights (EU) and with Unitary (EQ) Weights.

\begin{tabular}{|c|c|c|c|c|c|c|c|c|c|c|c|c|}
\hline Country & H 05 & H 06 & H 07 & H 08 & MD_EU 05 & MD_EU 06 & MD_EU 07 & MD_EU 08 & MD_EQ 05 & MD_EQ 06 & MD_EQ 07 & MD_EQ 08 \\
\hline AT & 9 & 9 & 7 & 8 & 3 & 5 & 5 & 11 & 5 & 6 & 8 & 10 \\
\hline $\mathrm{BE}$ & 14 & 13 & 13 & 12 & 11 & 11 & 10 & 7 & 11 & 9 & 9 & 7 \\
\hline$B G$ & & & & 24 & & & & 26 & & & & 25 \\
\hline $\mathrm{CY}$ & 15 & 14 & 15 & 15 & 20 & 20 & 22 & 18 & 19 & 20 & 20 & 18 \\
\hline$C Z$ & 3 & 2 & 1 & 1 & 17 & 17 & 15 & 14 & 16 & 16 & 16 & 15 \\
\hline DE & 8 & 10 & 14 & 13 & 6 & 13 & 12 & 13 & 7 & 13 & 12 & 11 \\
\hline DK & 6 & 6 & 6 & 5 & 4 & 4 & 4 & 3 & 3 & 4 & 4 & 5 \\
\hline EE & 16 & 16 & 21 & 20 & 16 & 14 & 14 & 8 & 20 & 17 & 17 & 12 \\
\hline ES & 23 & 22 & 22 & 21 & 10 & 9 & 9 & 10 & 13 & 11 & 10 & 9 \\
\hline $\mathrm{Fl}$ & 5 & 8 & 10 & 11 & 8 & 6 & 6 & 6 & 9 & 7 & 7 & 6 \\
\hline $\mathrm{FR}$ & 10 & 11 & 11 & & 12 & 12 & 13 & & 12 & 12 & 13 & \\
\hline GR & 21 & 24 & 24 & 23 & 19 & 19 & 18 & 15 & 18 & 18 & 18 & 16 \\
\hline $\mathrm{HU}$ & 12 & 15 & 8 & 9 & 22 & 22 & 23 & 24 & 22 & 22 & 24 & 24 \\
\hline IE & 22 & 18 & 17 & 14 & 7 & 10 & 11 & 16 & 6 & 10 & 11 & 14 \\
\hline IS & 2 & 1 & 2 & 2 & 13 & 8 & 8 & 5 & 8 & 5 & 5 & 3 \\
\hline IT & 17 & 21 & 23 & 18 & 14 & 15 & 17 & 17 & 14 & 14 & 15 & 17 \\
\hline LT & 24 & 23 & 20 & 22 & 24 & 23 & 21 & 21 & 24 & 23 & 22 & 21 \\
\hline LU & 13 & 12 & 12 & 10 & 1 & 1 & 1 & 2 & 1 & 1 & 2 & 2 \\
\hline LV & 19 & 25 & 25 & 26 & 25 & 25 & 25 & 23 & 25 & 25 & 25 & 23 \\
\hline NO & 4 & 3 & 9 & 4 & 5 & 3 & 2 & 1 & 4 & 3 & 1 & 1 \\
\hline PL & 25 & 19 & 16 & 16 & 23 & 24 & 24 & 22 & 23 & 24 & 23 & 22 \\
\hline PT & 20 & 17 & 18 & 17 & 18 & 18 & 20 & 20 & 17 & 19 & 19 & 19 \\
\hline RO & & & & 25 & & & & 25 & & & & 26 \\
\hline SE & 1 & 7 & 4 & 6 & 2 & 2 & 3 & 4 & 2 & 2 & 3 & 4 \\
\hline $\mathrm{SI}$ & 7 & 5 & 5 & 7 & 15 & 16 & 16 & 12 & 15 & 15 & 14 & 13 \\
\hline SK & 11 & 4 & 3 & 3 & 21 & 21 & 19 & 19 & 21 & 21 & 21 & 20 \\
\hline UK & 18 & 20 & 19 & 19 & 9 & 7 & 7 & 9 & 10 & 8 & 6 & 8 \\
\hline
\end{tabular}


Figure 1: Changes in the ranks in Material Deprivation (MD) with respect to Income Poverty $(\mathrm{H})$ among EU Member States in 2005 and 2006, with Eurobarometer Weights (EU) and with Unitary (EQ) Weights.

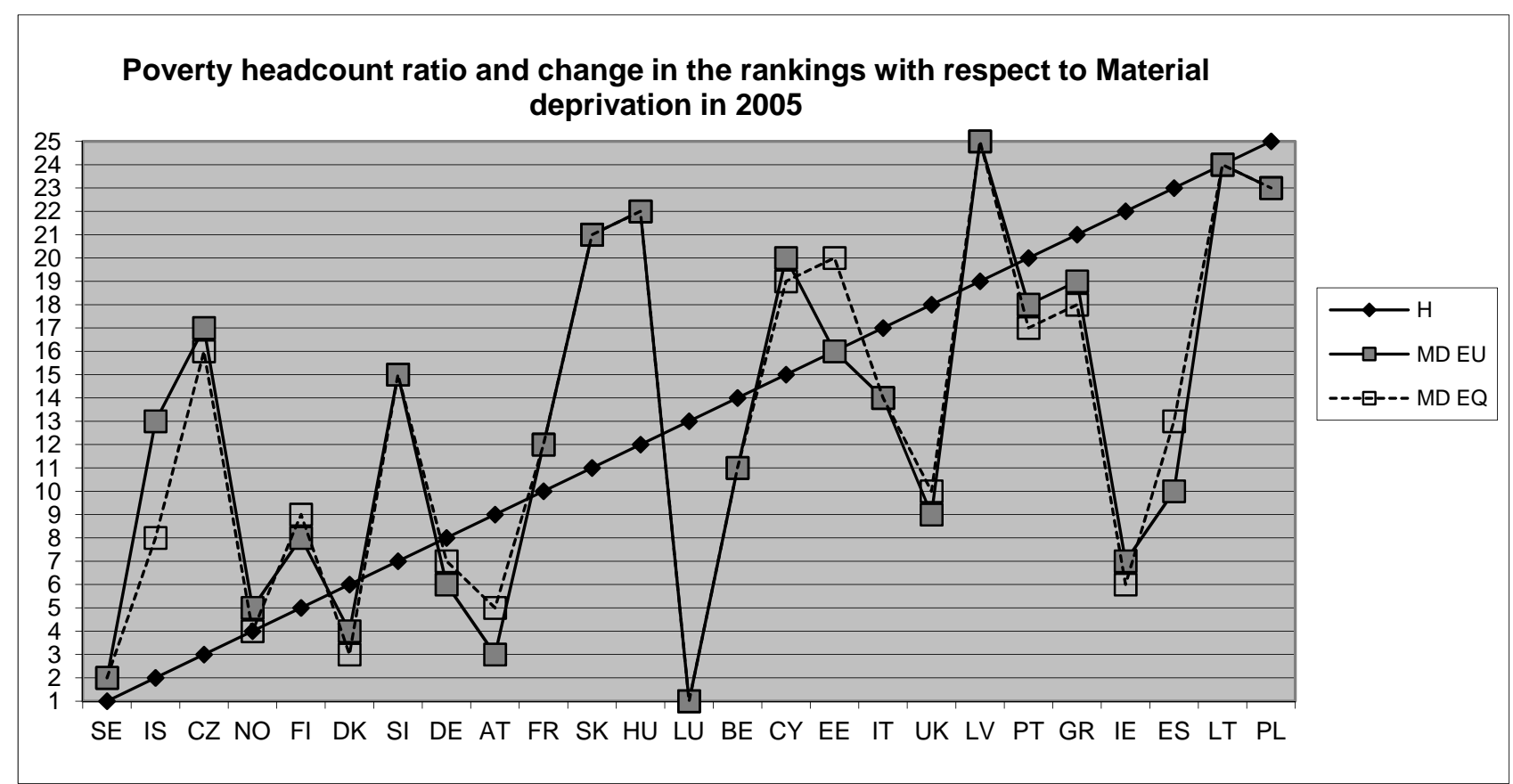

Poverty headcount ratio and change in the rankings with respect to Material deprivation in 2006

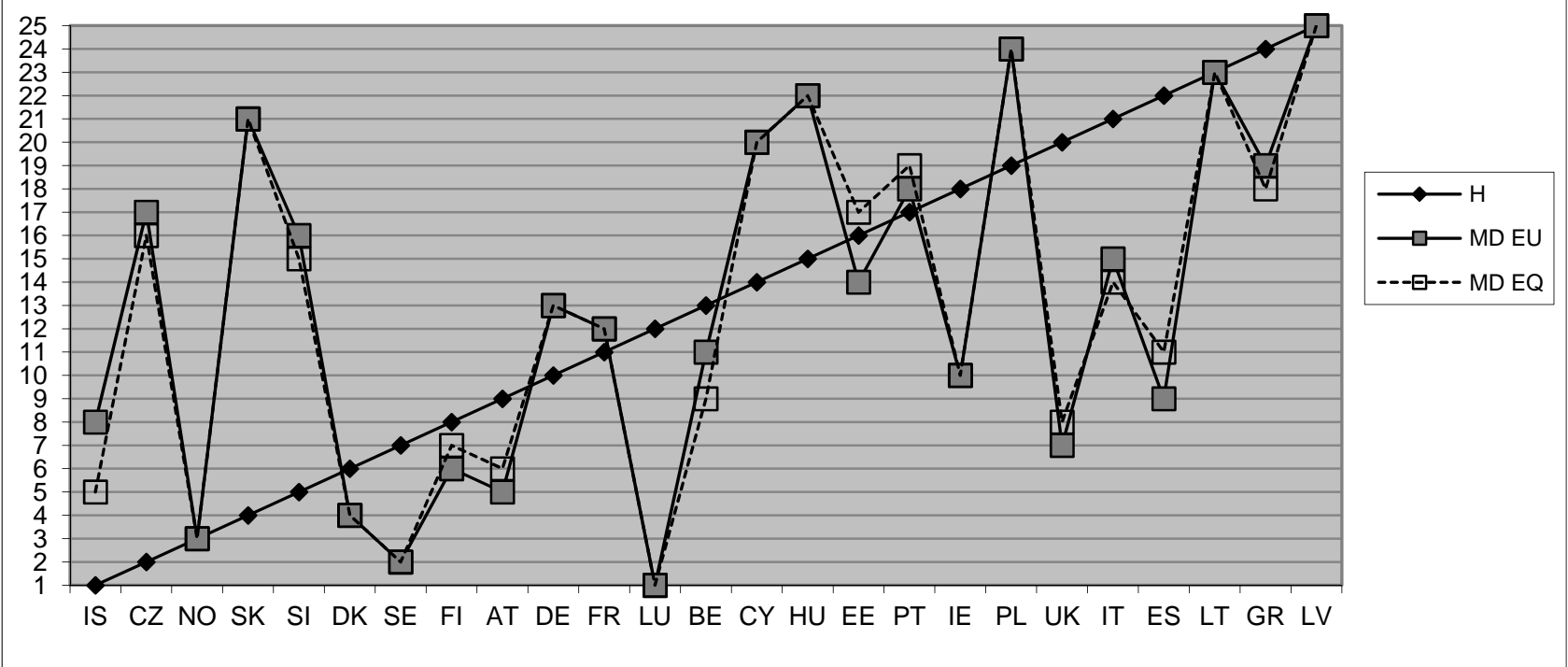


Figure 2: Changes in the ranks in Material Deprivation (MD) with respect to Income Poverty $(\mathrm{H})$ among EU Member States in 2007 and 2007, with Eurobarometer Weights (EU) and with Unitary (EQ) Weights.
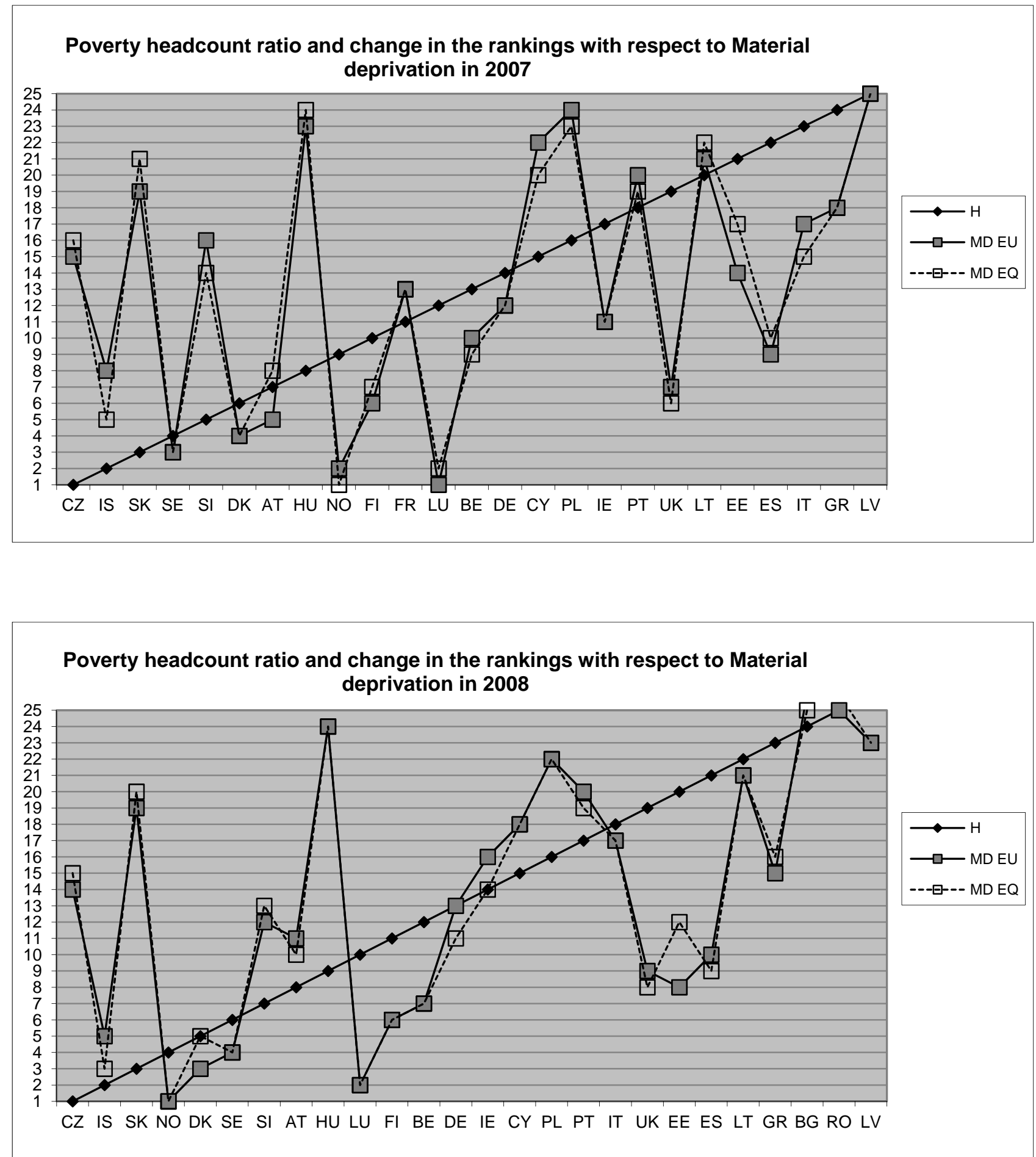